\title{
Solving an exponential Diophantine equation
}

\author{
by \\ Mario Huicochea (Guanajuato)
}

Introduction. Let $k>1, L>0, k, L \in \mathbb{Z}$, and let $p_{1}<\cdots<p_{L}$ be rational primes. We are going to show that if $\left(k, L, p_{1}, \ldots, p_{L}\right)$ satisfies

$$
k \prod_{i=1}^{L} p_{i}^{k-1}=\prod_{i=1}^{L} \frac{p_{i}^{k}-1}{p_{i}-1},
$$

then $k=2, L=2, p_{1}=2$ and $p_{2}=3$.

If we look carefully, the equation (1) with $k=2$ is a special case of the perfect numbers problem. In this paper, we solve (1) with elementary and analytic methods. The author was motivated by similar problems which appear in [G, Ch. B].

Preliminaries. We denote by $\omega(n)$ the number of different primes which divide $n$, and by $\pi(n)$ the number of primes which are at most $n$.

First note that if $\left(k, L, p_{1}, \ldots, p_{L}\right)$ solves (1) we have $L \neq 1$, because $\left(p_{1}, \frac{p_{1}^{k}-1}{p_{1}-1}\right)=1$.

Proposition 1. If $\left(k, L, p_{1}, \ldots, p_{L}\right)$ is a solution of $(1)$ and $2 \mid k$ then $k=2, L=2, p_{1}=2$ and $p_{2}=3$.

Proof. Let $k=2^{\alpha} r$ with $2 \nmid r$. For all $p_{i} \neq 2$ we have

$$
\frac{p_{i}^{k}-1}{p_{i}-1}=\frac{p_{i}^{r}-1}{p_{i}-1} \prod_{j=0}^{\alpha-1} \frac{p_{i}^{2^{j+1} r}-1}{p_{i}^{2^{j} r}-1} .
$$

Also, for all $0 \leq j \leq \alpha-1$ we have $2 \mid \frac{p_{i}^{2^{j+1} r}-1}{p_{i}^{j^{j} r}-1}$ so

$$
\frac{p_{i}^{k}-1}{p_{i}-1}=2^{\alpha} r_{i} \quad \text { with } r_{i} \in \mathbb{N} \text {. }
$$

2010 Mathematics Subject Classification: Primary 11D72; Secondary 11A41.

Key words and phrases: Diophantine equation, Euler's constant, prime decomposition. 
If $p_{1}>2$ then

$$
2^{\alpha} r \prod_{i=1}^{L} p_{i}^{k-1}=k \prod_{i=1}^{L} p_{i}^{k-1}=\prod_{i=1}^{L} \frac{p_{i}^{k}-1}{p_{i}-1}=2^{\alpha L} m \quad \text { with } m \in \mathbb{N},
$$

which contradicts $L \neq 1$.

Thus, we have $p_{1}=2$. Substituting $p_{1}=2$ in (1) gives

$$
2^{\alpha+k-1} r \prod_{i=2}^{L} p_{i}^{k-1}=k \prod_{i=1}^{L} p_{i}^{k-1}=\prod_{i=1}^{L} \frac{p_{i}^{k}-1}{p_{i}-1}=2^{\alpha(L-1)} \widetilde{m} \quad \text { with } \widetilde{m} \in \mathbb{N} .
$$

The last equality yields

$$
\alpha(L-1) \leq \alpha+k-1 .
$$

As $\left(k, L, p_{1}, \ldots, p_{L}\right)$ is a solution of $(1)$,

$$
k=\prod_{i=1}^{L} \frac{p_{i}^{k}-1}{p_{i}^{k-1}\left(p_{i}-1\right)}<\prod_{i=1}^{L} \frac{p_{i}}{p_{i}-1} \leq \prod_{i=1}^{L} \frac{i+1}{i}=L+1 .
$$

By (2) and (3),

$$
\alpha(L-1) \leq \alpha+k-1<\alpha+L .
$$

Suppose $L=2$. By (3), $k=2$ so

$$
4 p_{2}=\frac{2^{2}-1}{2-1} \frac{p_{2}^{2}-1}{p_{2}-1}=3 \frac{p_{2}^{2}-1}{p_{2}-1}
$$

which gives the solution $k=2, L=2, p_{1}=2$ and $p_{2}=3$.

If $L=3$, by (3), $k=2$, and if $L \geq 4$, by (4), $\alpha<L /(L-2) \leq 2$, thus from now on we assume $\alpha=1$.

If $L \geq 10$ we proceed as in (3):

$$
k=\prod_{i=1}^{L} \frac{p_{i}^{k}-1}{p_{i}^{k-1}\left(p_{i}-1\right)}<\prod_{i=1}^{L} \frac{p_{i}}{p_{i}-1} \leq \frac{2}{1} \cdot \frac{3}{2} \cdot \frac{5}{4} \prod_{i=5}^{L+1} \frac{i+1}{i}=\frac{3(L+2)}{4} .
$$

Thus, by (2) and (6), $\alpha(L-1) \leq \alpha+k-1<\alpha+(3 L+2) / 4$. In particular, $1=\alpha<\frac{3 L+2}{4(L-2)} \leq 1$, which is a contradiction.

It is sufficient to analyze those pairs which satisfy $2<L<10, k-1<L$, $2 \mid k$ and $4 \nmid k$. These conditions imply $k \in\{2,6\}$.

If $k=2$ and $L>2$ then, as in (5), $p_{2}=3$ and $2^{2} \cdot 3=\frac{2^{2}-1}{2-1} \cdot \frac{3^{2}-1}{3-1}$. So (1) is equivalent to

$$
\prod_{i=3}^{L} p_{i}=\prod_{i=3}^{L} \frac{p_{i}^{2}-1}{p_{i}-1}
$$

which is impossible since $p_{i}<\frac{p_{i}^{2}-1}{p_{i}-1}$. 
Finaly, if $k=6$ we have

$$
2^{6} \cdot 3 \prod_{i=2}^{L} p_{i}^{5}=\frac{2^{6}-1}{2-1} \prod_{i=2}^{L} \frac{p_{i}^{6}-1}{p_{i}-1}=3^{2} \cdot 7 \prod_{i=2}^{L} \frac{p_{i}^{6}-1}{p_{i}-1} .
$$

Thus, $p_{2}=3$ and $p_{i_{1}}=7$. This yields

$$
2^{6} \cdot 3^{6} \cdot 7^{5} \prod_{\substack{i=3 \\ i \neq i_{1}}}^{L} p_{i}^{5}=2^{5} \cdot 3^{3} \cdot 7^{2} \cdot 13 \cdot 19 \cdot 43 \prod_{\substack{i=3 \\ i \neq i_{1}}}^{L} \frac{p_{i}^{6}-1}{p_{i}-1} .
$$

Hence, there exists $1 \leq i_{2} \leq L$ such that $p_{i_{2}}=19$ and $2^{2} \mid \frac{19^{6}-1}{19-1}$. Consequently, by (7), $2^{7}$ divides the right hand side but not the left hand side of (1), which is impossible.

Lemma 1. If $\left(k, L, p_{1}, \ldots, p_{L}\right)$ is a solution of (1) with $p$ prime and $k=p^{\alpha}$ with $\alpha>0$ then $k=2, L=2, p_{1}=2$ and $p_{2}=3$.

Proof. By Proposition 1, if $p=2$ then $k=2, L=2, p_{1}=2$ and $p_{2}=3$.

Suppose $p \neq 2$. For each $p_{i}$ there exists $1 \leq j \leq L$ such that $p_{i} \mid \frac{p_{j}^{p^{\alpha}}-1}{p_{j}-1}$. By Fermat's Little Theorem,

$$
p_{i} \mid p_{j}^{p_{i}-1}-1 \text { and } p_{i} \mid p_{j}^{p^{\alpha}}-1 \text {. }
$$

If $p_{i} \mid p_{j}-1$ then $p_{j}=g p_{i}+1$ with $g \in \mathbb{N}$. So

$$
p_{i} \mid \sum_{l=1}^{p^{\alpha}}\left(\begin{array}{c}
p^{\alpha} \\
l
\end{array}\right)\left(g p_{i}\right)^{l-1} \quad \text { implies } p_{i} \mid p^{\alpha} \text { and } p_{i}=p .
$$

If $p_{i} \nmid p_{j}-1$ then, by $(8),\left(p^{\alpha}, p_{i}-1\right)>1$. In particular, $p \mid p_{i}-1$ so $p_{i}=\widetilde{g} p+1$ with $\widetilde{g} \in \mathbb{N}$. Since

$$
\frac{p_{i}^{k}-1}{p_{i}-1}=\sum_{l=1}^{p^{\alpha}}\left(\begin{array}{c}
p^{\alpha} \\
l
\end{array}\right)(\widetilde{g} p)^{l-1} \quad \text { and } \quad p^{\alpha} \mid \sum_{l=1}^{p^{\alpha}}\left(\begin{array}{c}
p^{\alpha} \\
l
\end{array}\right)(\widetilde{g} p)^{l-1},
$$

we conclude that

$$
p^{\alpha} \mid \frac{p_{i}^{k}-1}{p_{i}-1} \quad \text { but } \quad p^{\alpha+1} \nmid \frac{p_{i}^{k}-1}{p_{i}-1} .
$$

If $L=2$ we have two possibilities:

(*) $p \in\left\{p_{1}, p_{2}\right\}$ :

Without loss of generality suppose $p=p_{1}$. Then

$$
p^{\alpha+p^{\alpha}-1} p_{2}^{p^{\alpha}-1}=\frac{p^{p^{\alpha}}-1}{p-1} \frac{p_{2}^{p^{\alpha}}-1}{p_{2}-1} .
$$

Hence, $p^{p^{\alpha}-1} \mid \frac{p^{p^{\alpha}}-1}{p-1}$, which is impossible. 
$(* *) p \notin\left\{p_{1}, p_{2}\right\}:$

We know that $r_{i} p^{\alpha}=\frac{p_{i}^{k}-1}{p_{i}-1}$ with $p \nmid r_{i}$ for $i \in\{1,2\}$. Thus

$$
p^{\alpha} p_{1}^{p^{\alpha}} p_{2}^{p^{\alpha}}=\frac{p_{1}^{p^{\alpha}}-1}{p_{1}-1} \frac{p_{2}^{p^{\alpha}}-1}{p_{2}-1}=r_{1} r_{2} p^{2 \alpha}
$$

which contradicts the assumption $p \notin\left\{p_{1}, p_{2}\right\}$.

If $L=3$ then

$$
k=\prod_{i=1}^{3} \frac{p_{i}^{k}-1}{p_{i}^{k-1}\left(p_{i}-1\right)}<\prod_{i=1}^{3} \frac{p_{i}}{p_{i}-1} \leq \frac{3}{2} \cdot \frac{5}{4} \cdot \frac{7}{6}=\frac{105}{48}<3,
$$

which is impossible since $2 \nmid k$.

From now on we will assume $L>3$; then there are two primes $p_{i_{1}}, p_{i_{2}}$ which are not $p$. By the work above, $p^{\alpha} \mid \frac{p_{i_{1}}^{k}-1}{p_{i_{1}}-1}$ and $p^{\alpha} \mid \frac{p_{i_{2}}^{k}-1}{p_{i_{2}}-1}$; thus in (1) $p^{2 \alpha}$ divides the right hand side so there exists $1 \leq i_{0} \leq L$ such that $p_{i_{0}}=p$. With this observation, (1) implies

$$
p^{\alpha+p^{\alpha}-1} \prod_{\substack{i=1 \\ i \neq i_{0}}}^{L} p_{i}^{p^{\alpha}-1}=p^{\alpha(L-1)} \frac{p^{p^{\alpha}}-1}{p-1} \prod_{\substack{i=1 \\ i \neq i_{0}}}^{L} \frac{p_{i}^{p^{\alpha}}-1}{p^{\alpha}\left(p_{i}-1\right)}=p^{\alpha(L-1)} m
$$

with $m \in \mathbb{N}$ and $p \nmid m$. Hence, $\alpha+p^{\alpha}-1=\alpha(L-1)$. Also

$$
k=\prod_{i=1}^{L} \frac{p_{i}^{k}-1}{p_{i}^{k-1}\left(p_{i}-1\right)}<\prod_{i=1}^{L} \frac{p_{i}}{p_{i}-1} \leq \prod_{i=2}^{L+1} \frac{i+1}{i}=\frac{L+2}{2} .
$$

Thus,

$$
\alpha(L-1)=\alpha+k-1<\alpha+\frac{L+2}{2}-1=\alpha+L,
$$

in particular

$$
\alpha<\frac{L}{2(L-2)} \quad \text { and then } \quad \alpha<1 .
$$

This yields $\alpha=0$, which contradicts the assumption.

Lemma 2. If $\left(k, L, p_{1}, \ldots, p_{L}\right)$ solves (1) for $k$ with prime decomposition $k=\prod_{i=1}^{\omega(k)} q_{i}^{\alpha_{i}}$ where $\alpha_{i}>0$ and $2<q_{1}<\cdots<q_{\omega(k)}$, then $L<\omega(k)(k+1)$.

Proof. Define $Q_{i}=q_{i}^{\alpha_{i}}, \widetilde{Q}_{0}=1$,

$$
\mathcal{M}_{i}=\left\{\begin{array}{ll}
p_{j}: \text { there exists } p_{l} \text { such that } p_{j} \mid \frac{p_{l}^{\widetilde{Q}_{i}}-1}{p_{l}^{\widetilde{Q}_{i-1}}-1}
\end{array}\right\}
$$


and $\widetilde{Q}_{i}=\prod_{l=1}^{i} Q_{l}$. Note that

$$
\frac{p_{i}^{k}-1}{p_{i}-1}=\prod_{i=1}^{\omega(k)} \frac{p_{i}^{\widetilde{Q}_{i}}-1}{{\widetilde{Q}_{i-1}}_{i}} .
$$

Hence, there is $i_{0}$ such that $\left|\mathcal{M}_{i_{0}}\right| \geq L / \omega(k)$.

Note that if $p_{j} \mid \frac{\tilde{p}_{l} \widetilde{Q}_{i}-1}{\tilde{Q}_{l}{ }_{i-1}-1}$ then we have two possibilities:

(*) $p_{j} \mid p_{l}^{\widetilde{Q}_{i-1}}-1$ :

Let $w \in \mathbb{N}$ be such that $w p_{j}+1=p_{l}^{\widetilde{Q}_{i-1}}$. Then

$$
\frac{p_{l}^{\widetilde{Q}_{i}}-1}{p_{l}^{\widetilde{Q}_{i-1}}-1}=\frac{\left(w p_{j}+1\right)^{Q_{i}}-1}{w p_{j}}=\sum_{l=1}^{Q_{i}}\left(\begin{array}{c}
Q_{i} \\
l
\end{array}\right)\left(w p_{j}\right)^{l-1},
$$

thus $p_{j} \mid Q_{i}$ and $p_{j}=q_{i}$.

$$
(* *) p_{j} \nmid p_{l}^{\widetilde{Q}_{i-1}}-1 \text { : }
$$

By Fermat's Little Theorem, $p_{j} \mid\left(p_{l}^{\widetilde{Q}_{i-1}}\right)^{p_{j}-1}-1$ and $p_{j} \mid\left(p_{l}^{\widetilde{Q}_{i-1}}\right)^{Q_{i}}-1$. Thus, $\left(p_{j}-1, Q_{i}\right)>1$ and $q_{i} \mid p_{j}-1$. Let $p_{j}=v q_{i}+1$ with $v \in \mathbb{N}$. Then

$$
\frac{p_{j}^{Q_{i}}-1}{p_{j}-1}=\frac{\left(v q_{i}+1\right)^{Q_{i}}-1}{v q_{i}}=\sum_{l=1}^{Q_{i}}\left(\begin{array}{c}
Q_{i} \\
l
\end{array}\right)\left(v q_{i}\right)^{l-1} \text {, }
$$

and thereby $Q_{i} \mid \frac{p_{j}^{k}-1}{p_{j}-1}$.

In particular, for all $p_{j} \in \mathcal{M}_{i_{0}} \backslash\left\{q_{i_{0}}\right\}$ we have $Q_{i_{0}} \mid \frac{p_{j}^{k}-1}{p_{j}-1}$. Thus, $Q_{i_{0}}^{\left|M_{i_{0}}\right|-1}$ $=q_{i_{0}}^{\alpha_{i_{0}}\left(\left|M_{i_{0}}\right|-1\right)}$ divides the left hand side in (1), and the right hand side is equal to

$$
q_{i_{0}}^{\alpha_{0}} \frac{k}{q_{i_{0}}^{\alpha_{i_{0}}}} \prod_{\substack{i=1 \\ p_{i} \neq q_{i_{0}}}}^{L} p_{i}^{k-1} \quad \text { with } \alpha_{0} \in\left\{\alpha_{i_{0}}, \alpha_{i_{0}}+k-1\right\} .
$$

Moreover, if all $p_{i} \neq q_{i_{0}}$ then $\alpha_{0}=\alpha_{i_{0}}$, and if there is $j_{0}$ such that $p_{j_{0}}=q_{i_{0}}$ then $\alpha_{0}=\alpha_{i_{0}}+k-1$. Consequently, we have the inequality

$$
\alpha_{i_{0}}\left(\frac{L}{\omega(k)}-1\right) \leq \alpha_{i_{0}}\left(\left|\mathcal{M}_{i_{0}}\right|-1\right) \leq \alpha_{0} \leq \alpha_{i_{0}}+k-1 .
$$

Finally,

$$
L \leq \omega(k)\left(\frac{\alpha_{i_{0}}+k-1}{\alpha_{i_{0}}}+1\right) \leq \omega(k)(k+1) .
$$


Main theorem. We order the prime numbers as $P_{1}=2<P_{2}=3<$ $P_{3}=5<\cdots$.

THeOREM 1. If $k=\prod_{i=1}^{\omega(k)} q_{i}^{\alpha_{i}}$ with $\alpha_{i}>0$ and $2<q_{1}<\cdots<q_{\omega(k)}$ primes then there is no solution $\left(k, L, p_{1}, \ldots, p_{L}\right)$ of $(1)$.

Proof. Suppose that such a solution does exist. We have the inequality

$$
k=\prod_{i=1}^{L} \frac{p_{i}^{k}-1}{p_{i}^{k-1}\left(p_{i}-1\right)}<\prod_{i=1}^{L} \frac{p_{i}}{p_{i}-1} \leq \prod_{i=2}^{L+1} \frac{P_{i}}{P_{i}-1}=\frac{\prod_{i=1}^{L+1} \frac{P_{i}}{P_{i}-1}}{2},
$$

so by [RS],

$$
k<\frac{e^{C}}{2}\left(\log P_{L+1}\right)\left(1+\frac{1}{\log \log P_{L+1}}\right),
$$

where $e^{C}=1.78107 \ldots$ is Euler's constant.

By Lemma 1, $\omega(k)>1$ and thus $k \geq 15=3 \cdot 5$. Therefore

$$
14<\frac{e^{C}}{2}\left(\log P_{L+1}\right)\left(1+\frac{1}{\log \log P_{L+1}}\right) \quad \text { so } \quad P_{L+1}>17,
$$

thus $1+\frac{1}{\log \log P_{L+1}}>2$, which implies

$$
7<\frac{e^{C}}{2} \log P_{L+1}<\log P_{L+1} \quad \text { so } \quad e^{7}<P_{L+1} .
$$

We have $k=\prod_{i=1}^{\omega(k)} q_{i}^{\alpha_{i}} \geq 3^{\sum_{i=1}^{\omega(k)} \alpha_{i}} \geq 3^{\omega(k)}$ and Lemma 2 yields $L<$ $\omega(k)(k+1)$. By Table 1 and $[\mathrm{R}]\left(^{1}\right)$.

$$
\frac{P_{L+1}}{2+\log P_{L+1}} \leq \pi\left(P_{L+1}\right)=L+1<\omega(k)(k+1)+1 \leq \frac{(k+1) \log k}{\log 3}+1 .
$$

Our next step is to prove the following two claims.

Claim 1. If $x>4$ then

Proof. Define

$$
\frac{(x+1) \log x}{\log 3}+1<x^{3 / 2} .
$$

$$
f: \mathbb{R}^{+} \rightarrow \mathbb{R}, \quad f(x)=x^{3 / 2}-\frac{(x+1) \log x}{\log 3}-1 .
$$

Then

$$
f^{\prime}(x)=\frac{3}{2} x^{1 / 2}-\frac{\frac{x+1}{x}+\log x}{\log 3} \quad \text { and } \quad f^{\prime \prime}(x)=\frac{3}{4} x^{-1 / 2}-\frac{\frac{1}{x}-\frac{1}{x^{2}}}{\log 3} .
$$

Note that $x>\left(\frac{4}{3 \log 3}\right)^{2}$ implies $f^{\prime \prime}(x)>0$. Thus, if $x>4>\left(\frac{4}{3 \log 3}\right)^{2}$ we have $f^{\prime}(x)>f^{\prime}(4)>0$, and hence $f(x)>f(4)>0$.

$\left({ }^{1}\right)$ For all $x \geq 55$ prime, $\left[\mathrm{R}\right.$ gives the inequality $\frac{x}{2+\log x} \leq \pi(x)$; for $x<55$ prime, this follows from Table 1 . 
Claim 2. If $x>e^{6}$ then $x^{2}>8(1+\log x)^{5}$.

Proof. Let

$$
f: \mathbb{R}^{+} \rightarrow \mathbb{R}, \quad f(x)=x^{2}-8(1+\log x)^{5} .
$$

Then

$$
f^{\prime}(x)=2 x-40 \frac{(1+\log x)^{4}}{x} \quad \text { and } \quad f^{\prime \prime}(x)=2-\frac{40(1+\log x)^{3}(3-\log x)}{x^{2}} .
$$

Note that if $x>e^{3}$ then $f^{\prime \prime}(x)>2$. Hence, if $x>e^{6}$ then $f^{\prime}(x)>f^{\prime}\left(e^{6}\right)>0$, and so $f(x)>f\left(e^{6}\right)>0$.

Now we return to the proof of our theorem. Since $P_{L+1}>e^{7}$, Claim 1 and the inequalities (9), (11) give us

$$
\frac{P_{L+1}}{2+\log P_{L+1}}<\frac{(k+1) \log k}{\log 3}+1<k^{3 / 2}
$$

and

$$
k^{3 / 2}<\left(\frac{e^{C}}{2}\left(\log P_{L+1}\right)\left(1+\frac{1}{\log \log P_{L+1}}\right)\right)^{3 / 2}<\left(e^{C} \log P_{L+1}\right)^{3 / 2} .
$$

In particular

$$
\begin{aligned}
P_{L+1}^{2} & <\left(2+\log P_{L+1}\right)^{2}\left(e^{C} \log P_{L+1}\right)^{3}<e^{3 C}\left(1+\log P_{L+1}\right)^{5} \\
& <8\left(1+\log P_{L+1}\right)^{5} .
\end{aligned}
$$

But this contradicts Claim 2, showing there is no such solution.

Table 1

\begin{tabular}{rrc}
\hline$P_{L}$ & $L$ & $\frac{P_{L}}{2+\log P_{L}} \approx$ \\
\hline 2 & 1 & 0.74262 \\
3 & 2 & 0.96817 \\
5 & 3 & 1.38525 \\
7 & 4 & 1.77398 \\
11 & 5 & 2.50911 \\
13 & 6 & 2.84778 \\
17 & 7 & 3.51732 \\
19 & 8 & 3.84270 \\
23 & 9 & 4.47863 \\
29 & 10 & 5.40309 \\
31 & 11 & 5.70483 \\
37 & 12 & 6.59428 \\
41 & 13 & 7.17589 \\
43 & 14 & 7.46372 \\
47 & 15 & 8.03398 \\
53 & 16 & 8.87728 \\
\hline
\end{tabular}




\section{References}

[G] R. K. Guy, Unsolved Problems in Number Theory, Springer, New York, 2004.

[R] J. B. Rosser, Explicit bounds for some functions of prime numbers, Amer. J. Math. 63 (1941), 211-232.

[RS] J. B. Rosser and L. Schoenfeld, Approximate formulas for some functions of prime numbers, Illinois J. Math. 6 (1962), 64-94.

Mario Huicochea

Centro de Investigación en Matemáticas (CIMAT)

Jalisco S/N, Col. Valenciana

CP 36240 Guanajuato, Gto, México

E-mail:dym@cimat.mx

Received on 19.2.2009

and in revised form on 18.6.2010 